\title{
Computational study of tungsten sputtering by nitrogen
}

\section{Lyashenko, A.}

2020-12-15

Lyashenko , A , Safi , E , Polvi , J , Djurabekova , F \& Nordlund, K 2020 , ' Computational

study of tungsten sputtering by nitrogen ' , Journal of Nuclear Materials , vol. 542 , 152465 . https://doi.org/10.1016/j.

http://hdl.handle.net/10138/322868

https://doi.org/10.1016/j.jnucmat.2020.152465

cc_by_nc_nd

acceptedVersion

Downloaded from Helda, University of Helsinki institutional repository.

This is an electronic reprint of the original article.

This reprint may differ from the original in pagination and typographic detail.

Please cite the original version. 


\title{
Computational study of tungsten sputtering by nitrogen
}

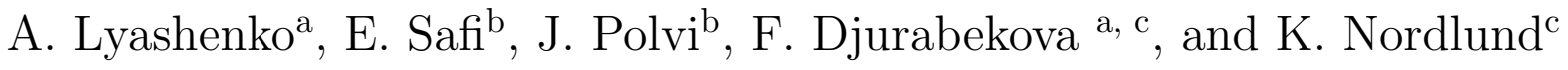 \\ ${ }^{a}$ National Research Nuclear University MEPhI, 115409 Moscow, Russia \\ ${ }^{\mathrm{b}}$ Department of Physics, PB 43, 00014 University of Helsinki, Finland \\ ${ }^{\text {c} D e p a r t m e n t ~ o f ~ P h y s i c s ~ a n d ~ H e l s i n k i ~ I n s t i t u t e ~ o f ~ P h y s i c s, ~ P B ~ 43, ~} 00014$ \\ University of Helsinki, Finland
}

\section{Highlights}

- A newly developed W-N potential based on Tersoff-Brenner formalism was used

- $\mathrm{N}$ accumulation reduces $\mathrm{W}$ sputtering yield by almost an order of magnitude

- Approximately half of sputtered W atoms leave the surface as a WN molecule

- Nitrogen saturation occurs by the growth of the $\mathrm{N}$ sputtering yield with the fluence

- Nitrogen reflection coefficient remains constant during the accumulation

\section{Abstract}

Gaseous nitrogen is planned to be used as a seeding species to control the power flux in future fusion reactors with ITER-like divertors. Nitrogen interacts with the first wall materials, particularly with tungsten, leading to sputtering and changes of chemical composition of the material. We use the molecular dynamics methods with a recently developed WN potential to analyze the mechanisms leading to these modifications. We performed the simulations of cumulative nitrogen irradiation runs of tungsten surface. The sputtering yields obtained in our cumulative runs are in good agreement with experimental data. We observe the decrease of the tungsten sputtering yield with nitrogen accumulation and determine the reasons for the observed trend. The cluster analysis reveals the composition of the sputtered particles, suggesting the swift chemical sputtering process that occurs under the prolonged nitrogen irradiation of tungsten. We also observe 
and analyze the nitrogen saturation in the temperature range below the thermal stability limit.

Keywords: tungsten - nitrogen interaction, tungsten sputtering yield, tungsten nitride, nitrogen retention in tungsten, impurity seeded plasma, plasma surface interactions, molecular dynamics

\section{Introduction}

One of the most important problems of thermonuclear power engineering is lowering the extremely high power load on plasma-facing components (PFCs) in tokamaks. Quasistationary heat fluxes up to $20 \frac{\mathrm{MW}}{\mathrm{m}^{2}}$ are expected on the PFCs during ITER operation [1]. These fluxes are almost at the limit of the possibilities of modern cooling systems and materials. Also, at abnormal operation conditions, during plasma disruptions, heat fluxes up to several $\frac{\mathrm{GW}}{\mathrm{m}^{2}}$ are expected for a few ms [2]. Noble gases or nitrogen are planned to be added as seeding species in the scrape-off layer (SOL) [3-5]. It reduces the power load on the PFCs because the gases uniformly reradiate heat fluxes to the walls. A more even distribution of the fluxes allows reducing the load on the divertor to acceptable values. $[6,7]$. On the other hand, the seeding species may help keep the plasma hot and improve plasma confinement. Experimental results show that nitrogen can do both, and it is better for SOL plasma cooling than noble gases. Hence nitrogen is planned to be used in future operations, particularly in ITER $[6,7]$.

However, nitrogen interaction with the PFCs leads to erosion and changes in the physical properties of the materials $[7,8]$, since nitrogen atoms can form chemical bonds with the PFC materials, unlike noble gases. Mainly, N atoms interact with the tungsten (W) divertor, forming WN compounds. Thermal stability of WN is a critical issue concerning the condition of a thermonuclear reactor. A sharp increase of the first wall temperature may lead to a sudden release of large amounts of nitrogen from the material, affecting the plasma operation. Unfortunately, there is no clear consensus about the temperature of thermal decomposition of $\mathrm{WN}$ in the literature.

The first studies of this subject were performed with the WN powder, which was produced chemically. In these works [9-11], the tungsten nitride decomposition was found to occur at approximately $870 \mathrm{~K}$ by the following chemical reaction: $2 \mathrm{WN}=\mathrm{W}_{2} \mathrm{~N}+\frac{1}{2} \mathrm{~N}_{2}$ [9-11]. However, the calculations performed by ThermoCalcTM [12] predicted the decomposition of mononitride at $600 \mathrm{~K}$. The authors also measured $\mathrm{N}$ areal density as a function of target temperature by nuclear reaction analysis (NRA) and confirmed decrease 
of retention in the temperature range between 600 and $750 \mathrm{~K}$ [8]. Another study investigated thermal desorption spectra (TDS) of tungsten samples with previously implanted nitrogen and showed that most of the nitrogen outgases at approximately $1200 \mathrm{~K}$ [13].

Finally in Ref. [14], the authors attempted to resolve the apparent contradiction. By performing another thermal desorption analysis, they demonstrated that nitrogen accumulation in tungsten exhibits a complex dependency on material temperature. Nitrogen retention decreases steadily with increasing tungsten temperature. On the other hand, while heating the N-implanted W sample, a diffusive loss of $\mathrm{N}$ was observed only above 800 K. They also showed that nitrogen decomposition requires significant time to appear [14] due to low diffusivity of nitrogen [15], [16]. Therefore, the thermal desorption spectra (TDS) showed decomposition only at $970 \mathrm{~K}$, and the authors recalculate it with respect to characteristic diffusion time from the upper atomic layers to the surface. In [13], only the TDS results were presented, and the authors heated samples faster than in [14]. These factors may be an explanation of the higher desorption temperature in [13]. Summarizing the literature findings, WN is thermally stable at least below $800 \mathrm{~K}$, and hence, may exist in the cooler parts of a thermonuclear reactor divertor. Moreover, physics of the WN compounds are also of interest for industrial applications. For example, tungsten mononitride films are used in microelectronics.

Nitrogen was seen to be stored in a thin layer on the tungsten surface during the exposure to nitrogen plasma. The layer is limited to a few nanometers, and the depth depends on incident energy. The bombarding $\mathrm{N}$ forms mononitride (WN) phases in tungsten $[7,8,13,14,17,18]$. Schmid et al. [8] experimentally investigated sputtering of tungsten with $\mathrm{N}$ ions of three energies: 300, 400, and $500 \mathrm{eV}$ and showed that nitrogen accumulation decreases tungsten sputtering yield. They also used the binary collision approximation (BCA) [19] to explain the result. Kallenbach et al. [7] presented BCA calculations of nitrogen mean projected ranges in tungsten at $20 \mathrm{eV}, 100 \mathrm{eV}$, and $500 \mathrm{eV}$. The same BCA approach was used to study the nitrogen saturation in tungsten [14].

As the name suggests, binary collision approximation considers only two body interactions. All collisions are considered elastic, and atoms trajectories are calculated based on the law of conservation of energy and momentum using chosen interaction potential. A universal screened Coulomb scattering potential is used in TRIM based BCA codes, the results of which will be compared with ours further. The resulting trajectories are a sequence of straight lines corresponding to the asymptotes between the collisions. Surface binding energy is one of the most significant adjustable parameters for sputtering yield calculations in these codes. It determines whether an atom coming to the surface from the bulk has enough energy to leave the material. Despite the beneficial computational efficiency of the BCA approach, the simplified interpretation of atomic collisions limits the value of physical insights, which the method can provide. In particular, binary collision approximation often can not accurately describe sputtering yield at energies close 
to the sputtering threshold, when many-body effects begin to play a significant role [19]. The study of this energy range is crucial for thermonuclear fusion. Molecular dynamics, used in the current article, is based on direct integration of equations of motions of all atoms in the system and, hence, can deliver more accurate information about the sputtering process even at low energies. One of the essential strengths of MD is the ability to simulate the surface changes during the bombardment, including chemical processes. The latter relies on the existence of a suitable interatomic potential. Nevertheless, it should be noted that MD calculations are computationally more demanding.

Polvi et al. has recently developed a well-suited potential for describing the tungstennitride (WN) system [20]. It is an analytical bond-order potential (ABOP) type, also known as the Tersoff - Brenner potential [21,22]. The ABOP potential functional form parameters for the WN system were fitted to a density functional theory (DFT) calculations database.

The potential showed good agreement with all data except elastic constants [20]. The fitting process in general is considered in detail in $[23,24]$. The potential accurately describes nitride phases and $\mathrm{N}$ interstitials binding energy. Therefore, the potential allows predicting the surface changes during nitrogen bombardment, including chemically driven processes.

In this work, we employ the newly developed WN ABOP potential [20] to study the sputtering of tungsten (W) by monoatomic nitrogen (N) using a classical MD method. We simulate cumulatively the collision cascades initiated by the incident $\mathrm{N}$ ions with energies ranging from $10 \mathrm{eV}$ to $1000 \mathrm{eV}$ and ambient temperatures 300 and $600 \mathrm{~K}$. In this article, we show only the results for $300 \mathrm{~K}$, since no significant difference was found in the cumulative simulations between the two temperature simulation series. We study the $\mathrm{N}$ accumulation in the material and its influence on the sputtering mechanisms. We also perform the cluster analysis of the sputtered particles to obtain the composition of sputtered molecules. The simulation results are compared with existing experimental and BCA data $[7,8]$.

\section{Simulation setup}

The initial tungsten sample consists of 3000 atoms organized in a body-centered cubic (bcc) lattice. The bulk is a $27 \times 27 \times 67 \AA$ single crystal. We apply periodic boundary conditions to the lateral faces of the sample. An infinitely large surface is simulated this way. The ( $\left.\begin{array}{lll}0 & 0 & 1\end{array}\right)$ crystal direction normal to the z-axis is opened to simulate a surface in vacuum. Three atom layers at the bottom of sample are fixed. This approach allows avoiding a movement of the bulk as a whole in the z-direction. Before the irradiation, the bulk was relaxed under the desired temperature.

We performed the relaxation in the following way. At first, we constructed a $\mathrm{W}$ sim- 
ulation cell with the bcc structure and lattice parameter $a=3.160 \AA$ that corresponds to the room temperature value in the chosen interatomic potential [20]. Then periodic boundary conditions were set. We relaxed the system in the NPT ensemble by applying the Berendsen barostat and thermostat [25]. After that, we removed the periodic boundaries in the z-direction and relaxed the system again in NVT ensemble for 100 ps until the total energy reached the minimum value. This way, we generated a stable surface with the equilibrium lattice parameter, corresponding to the desired temperature.

Nitrogen atoms are launched from a height of $5 \AA$ above the surface at the $10^{\circ}$ angle with respect to the z-direction. We use tilt to avoid possible channeling effect during the bombardment [26]. The surface level is defined by the highest $\mathrm{z}$ coordinate of the bulk atoms. A new surface level is checked after each recoil because it changes due to sputtering and nitrogen accumulation during the bombardment. The azimuthal angle of ion impact is chosen randomly. We use random shifting of the sample in $\mathrm{x}, \mathrm{y}$ directions (taking periodic boundaries into account) to simulate uniform flux after each impact [27].

Molecular dynamics code PARCAS is used for the simulation [28]. We simulate cumulative tungsten sputtering by atomic nitrogen with energies from 10 to $1000 \mathrm{eV}$ at the sample temperatures 300 and $600 \mathrm{~K}$. Each cumulative run simulates 7 ps of experimental time for most cases. We have performed about several thousand impacts for each case. Due to high computational costs, it is impossible to simulate irradiation with the same value of fluxes as in the experiment. The latter amounted to $(3-5) \cdot 10^{19} \mathrm{~m}^{2} \mathrm{~s}^{-1}$ depending on the ion energies [8], while in the model the flux is as high as $10^{28} \mathrm{~m}^{2} \mathrm{~s}^{-1}$. Taking into account the average number of simulated collisions, we model a fluence of the order of $10^{19} \mathrm{~m}^{-2}$. Maximum fluence is simulated for an energy of $10 \mathrm{eV}: 8 \cdot 10^{19} \mathrm{~m}^{-2}$. The fluence varies in the range $1.6-4 \cdot 10^{19} \mathrm{~m}^{-2} \mathrm{eV}$ for the other energies. The small size of the simulated sample and periodic boundary conditions may lead to an overestimation of nitrogen concentration in the material. However, experimental measurements show than nitrogen diffusion is negligible at studied temperatures for the total modeled time $[15,16]$.

\section{Results}

\subsection{The tungsten sputtering yield}

The first step of our study is to compare the new WN potential calculations of W sputtering by $\mathrm{N}\left(Y_{W}\right)$ with the data from the literature. Instead of performing statistical runs, we analyze the evolution of sputtering yield with the number of incoming $\mathrm{N}$ ions, i.e. $Y_{W}=\frac{\sum N_{W}}{\sum N_{\text {nitrogen }}}$, where $\sum N_{W}$ is the number of sputtered tungsten atoms and $\sum N_{\text {nitrogen }}$ is the number of incident nitrogen atoms. The dependence of $\mathrm{W}$ sputtering yield on the number of the incident ions is illustrated in fig. 1. As already mentioned, there are 2 sources that can be used to validate the present results: various BCA codes 
calculations $[7,8,14]$, and the experiment performed by Schmid et al. [8]. Our MD simulations show that the tungsten sputtering coefficient $\left(Y_{W}\right)$ decreases by almost an order of magnitude with cumulative runs. The more projectiles impact on the sample, the lower the tungsten sputtering $\left(Y_{W}\right)$ becomes. This decrease gradually slows down, and eventually, the tungsten sputtering coefficient comes to a certain value for each projectile energy. The increase in the number of impacts causes only some fluctuations of the sputtering yield around this value. The reason for the changes in sputtering yield is the accumulation of nitrogen in the surface. We discuss this issue in more detail below.

The sputtering yields from the literature strongly depend on the chosen BCA code. Schmid et al. [8] applied code TRIDYN [38], that includes static and dynamic approaches. TRIDYN is based on TRIM code, which implements the static method. For this reason, TRIDYN is also called dynamic TRIM in the literature $[7,8]$. Unlike the dynamic approaches, static BCA calculations do not take into account material changes and hence accumulation of nitrogen during the bombardment. As a result, the TRIM sputtering yield is much higher than dynamic TRIDYN calculations and experimental results. Molecular dynamics allows following the gradual change in the coefficient from the pure $\mathrm{W}$ sample (TRIM results) to the nitrogen saturated surface case (TRIDYN in dynamic mode and experiment). Schmid et al. [8] set surface binding energy to $8.86 \mathrm{eV}$, which corresponds to pure $\mathrm{W}$ heat of sublimation. The values of other important parameters are not indicated in the article. The default TRYDIN (and TRIM) values were probably used: cutoff energy is equal to surface binding energy, and bulk binding energy the authors set to be zero. The composition of the incident particles was set in accordance with the experimental data: $5 \% N^{3+}, 85 \% N^{2+}$ and $10 \% N^{+}$. The authors manually limit nitrogen concentration in the material by $50 \%$ in the dynamic regime. We averaged the tungsten sputtering coefficients over the first 1000 cumulative runs, which are shown as the first two points in the fig. 1. This part of the modeling can be treated as a pure material study because at that point there are not enough nitrogen atoms to influence the sputtering yield. Hence we compare these values with the TRIM calculations, fig. 2. Then we averaged the last part of the modeling, where $Y_{W}$ reaches the constant value (the last 3 points in the fig. 1). These values correspond to the nitrogen saturation stage and can be compared directly with dynamic TRIDYN and experimental data, fig. 3. Both results are in good agreement with our MD calculations. At $300 \mathrm{eV}$ energy, the MD results agree with the experimental result much better than dynamic TRIDYN.

Let us now consider the tungsten sputtering dependence on nitrogen fluence. Schmid et al. showed that $Y_{W}$ decreasing with the $\mathrm{N}$ ion fluence due to the decrease of tungsten concentration on the surface. Dynamic BCA results support this conclusion. However, TRIDYN can take into account only compositional changes of the surface: tungsten surface binding energy remains constant during the simulations. Molecular dynamics has an opportunity to study surface changes in greater detail. Our calculations showed that 
nitrogen accumulation increases tungsten cohesive energy on the surface by tenths of an eV per atom, fig. 4. By definition, the cohesive energy of a solid is the energy required to break the atoms of the solid into isolated atoms. This factor can be another reason for the $Y_{W}$ decreasing along with the compositional one.

\subsection{Nitrogen accumulation in tungsten. The saturation}

This section describes nitrogen accumulation in tungsten and specifically the saturation of the sample surface with the incident nitrogen atoms. Fig. 5 illustrates nitrogen accumulation dependence on the number of incident atoms for various energies. The amount of nitrogen retained in tungsten grows gradually. However, the slope of the growth decreases with the number of incident ions, indicating a saturated amount of nitrogen, which is trapped within tungsten at given incident energy. The lower the energy, the faster this saturation is reached. With an increase of incident energy, the nitrogen penetrates deeper into tungsten, allowing for more nitrogen to be accommodated within the tungsten lattice. This is also seen by the identical initial growth rate of nitrogen retained in the lattice at all incident energies. The lower the incident energy, the faster the growth rate deviates from the initial value. We do not observe the saturation in the 5 even after 3-4 thousand impacts, but the $\mathrm{N}$ retention slows significantly for the lowest energies $(10 \mathrm{eV}$ and $20 \mathrm{eV})$. Further we continue cumulative simulation for $10 \mathrm{eV}$ energy to reach the saturation, fig. 7 . In this case, nitrogen accumulation slows noticeably after 500-1000 runs. In contrast to the $10 \mathrm{eV}$ bombardment, there was no slowdown in nitrogen accumulation at $100 \mathrm{eV}$ and higher energies (after 2-5 thousand of impacts). At the highest energies in the simulated range (500 and $1000 \mathrm{eV}$ ), some of the ions left the simulation box, which was rather small for these energies. However, these ions do not affect the overall trend of reaching the retained nitrogen's saturation level as they would stop far below the surface.

The process of nitrogen storage occurs as follows. At the beginning of the bombardment, nitrogen is randomly distributed in the simulation cell according to the range profile. Hence the highest concentration of nitrogen is near the mean penetration depth $([-20,0]$ $\AA$ interval in fig. 4). This depth and the shape of the nitrogen distribution are defined by the incident energy. The depth of the penetration is roughly proportional to the square root of the energy. At the same time, the higher the incident energy increases, the wider the distribution becomes. This means that at high energies, nitrogen accumulates more evenly in the material. Nevertheless, the layer where the nitrogen concentration exceeds that of tungsten appears for all simulated energies (10-1000 eV). At low energies, an oversaturated layer forms faster due to the narrow peaks of nitrogen concentration.

During accumulation, excess nitrogen from this oversaturated layer $([-20,0] \AA$ interval in fig. 4) starts pushing the tungsten atoms towards the surface, leading to the swelling 
of the tungsten lattice ([0,15] $\AA$ interval in the fig. 4). The swelling rate depends on how narrow the nitrogen concentration peak is. In general, the lower the energy, the faster the swelling progresses. For example, such layer appears, and swelling begins for the $20 \mathrm{eV}$ case after approximately 50 accumulated nitrogen atoms; for the $100 \mathrm{eV}$ case after 200 atoms, and for the $500 \mathrm{eV}$ case after 500 atoms. The surface layer of the sample increased by $1.5 \mathrm{~nm}$ during 3379 runs of $300 \mathrm{eV}$ bombardment, fig. 4 and fig. 6. The process probably does not reflect the experiment. At least we cannot rely on this result because of the significant difference between the elastic constant of the Polvi et al. potential and the reference DFT modeling values.

Basically, tungsten cohesive energy increases in the material during nitrogen accumulation. This happens even if the nitrogen concentration is low. The cohesive energy increases noticeably even at a concentration of less than $2 \mathrm{~N}$ to $10 \mathrm{~W}$ (in the depth of the sample), fig. 4. Nevertheless, if the nitrogen concentration is too high, then the energy may decrease a little. It occurs near the mean stopping range of incoming atoms, see fig. 4 about -10 A from the surface. At this point, the concentration of nitrogen atoms significantly exceeds that of the WN crystal (1:1). This is not an entirely correct parameter for comparison since the crystal does not form within the framework of our simulation. However, even in spite of the apparent nonequilibrium state of the system: the cohesive energy of tungsten atoms is maximal precisely near the ratio of $N_{W}$ to $N_{N} 1$ to 1 . Thus, for $300 \mathrm{eV}$ energy, the maximum is reached approximately at $0.9 \frac{N_{N}}{N_{W}}$ ratio, fig. 4 . Deviations from the equilibrium value can be explained by a large flux of incident particles (many orders of magnitude higher than the experimental ones). The incident particles constantly destroy the bonds between the atoms of the sample, preventing the formation of a stable structure. Nitrogen destroys existing tungsten bonds near the stopping point and forms $\mathrm{N}-\mathrm{N}$ bonds instead of $\mathrm{W}-\mathrm{N}$ when there is too much nitrogen in this area. As a result, the tungsten atoms are less attached to the material than in the rest of the sample.

When the accumulation of nitrogen in the material begins to slow down noticeably, characteristic changes occur with the surface. The highest energy we observed such changes is $100 \mathrm{eV}$ (and all the smaller ones). However, the saturation was only simulated for $10 \mathrm{eV}$. Surface roughness increases strongly at the point when the slowdown of $\mathrm{N}$ retention becomes significant. There are many nitrogen and $\mathrm{WN}_{\mathrm{x}}$ molecules on the top of the surface at that moment. The nitrogen concentration exceeds the tungsten one several times, and the roughness contributes to the saturation. This is discussed below in more detail. Fig. 6 represents the difference between the sample before and after $\mathrm{N}$ retention slowdown begins. For $300 \mathrm{eV}$ energy, the retention speed does not change significantly during the simulation (right side of fig. 6). On the contrary, the roughness is visible at $100 \mathrm{eV}$ energy bombardment (left side of fig. 6).

We achieved the saturation after approximately 4-5 thousand incident nitrogen ions for $10 \mathrm{eV}$ energy, fig. 7. To increase the computational efficiency, we increased the nitrogen 
flux after about 1200 runs by decreasing the simulation time of every impact from 7 ps to 2 ps. The saturation of nitrogen in tungsten can be described in terms of sputtering and reflection yields. We found that the reflection coefficient $\left(R_{N}\right)$ is approximately constant during the entire simulations. Furthermore, it does not depend on the energy of the incoming particle. A value of the coefficient is approximately 0.3 for all cases. The value varies slightly between 0.22 and 0.32 . On the other hand, the nitrogen sputtering yield $\left(\mathrm{Y}_{\mathrm{N}}\right)$ grows with an increase of the incident ions until it saturates at the value $Y_{N} \approx 1$. The amount of incoming and sputtered nitrogen becomes equal.

The growth of the nitrogen sputtering yield $\left(\mathrm{Y}_{\mathrm{N}}\right)$ during the cumulative bombardment can be explained by the growth of the nitrogen concentration on the tungsten surface. At the same time, the part of energy transferred in a single collision is much higher in the $\mathrm{N}-\mathrm{N}$ collision than in the $\mathrm{N}-\mathrm{W}$ collisions in accordance with the formula:

$$
E_{\text {max }}=\frac{4 M_{1} M_{2}}{\left(M_{1}+M_{2}\right)^{2}} E_{\text {inc }} \cos ^{2} \theta
$$

Here $M_{1}$ is a projectile mass, and $M_{2}$ is a mass of target atoms. $E_{i n c}$ stands for the incident energy, and $\theta$ is the angle between the momenta of the colliding particles.

Previously, binary collision approximation (BCA) has been used to study the nitrogen saturation in the tungsten sample [14], where energies of incoming ions were simulated: 500 and $2500 \mathrm{eV}$. The authors developed a special technique for comparing the profiles achieved by the BCA calculations with the x-ray photoelectron spectroscopy (XPS) measurements. The saturation profile of nitrogen achieved with the $2500 \mathrm{eV}$ energy was in a good agreement with the experimental data. On the contrary, the $500 \mathrm{eV}$ profiles differed significantly from the XPS results. Also, the nitrogen concentration in the sample exceeded the $50 \%$ limit after the simulation was completed. To deal with this problem, the nitrogen limit in the material set manually. It allowed achieving much better agreement with the experimental profiles was achieved. These results showed that some essential N retention processes can not be modeled by the BCA method at the low energies. Probably they also influence the other parameters of the WN system. The authors offered several possible explanations of the $500 \mathrm{eV}$ case disagreement with the experiment. First is the ion-induced diffusion of the excess nitrogen towards the surface and its possible recombination to a $\mathrm{N}_{2}$ molecule. Second, nitrogen prefers to make bonds in the unsaturated layer closer to the surface, and then it could be easily sputtered.

During our study, we observed the intense growth of the $\mathrm{N}$ concentration at the very surface, when the $\mathrm{N}$ retention starts to slow down. We also observed the roughness of the surface. There are many broken WN bonds inside the material and on the surface. Mononitride and nitrogen molecules and atoms bond to the sample weakly. A large quantity of nitrogen is sputtered in a molecular state (more than a half in the saturation case), fig. 8. The mechanism of sputtering is discussed in the 3.3.

We also estimate the influence of temperature on the sputtering yields. We simulated 
cumulative bombardment at two temperatures: 300 and $600 \mathrm{~K}$. Usually, temperature significantly influences physical sputtering only at temperatures close to the melting point. However, temperature generally sharply increases chemical sputtering. This happens because of the acceleration of the chemical reactions with increasing temperature. Also, temperature rise increases diffusion, which also leads to the higher sputtering yield, if volatile molecules are produced during the bombardment [29]. Nevertheless, we did not find a statistically significant difference in the resulting sputtering yields. Even the division of sputtered particles did not change the result notably. It also means that the current MD approach does not predict nitride decomposition at $600 \mathrm{~K}$. We have stopped the simulations for the $600 \mathrm{~K}$ ambient temperature after 1000-1500 impacts. All of the presented results are made for the $300 \mathrm{~K}$ case.

\subsection{Analysis of the sputtered particles}

Tungsten, nitrogen, and their chemical compounds are sputtered during the cumulative bombardment. Fig. 9 presents the dependence of the tungsten and nitrogen yields on the incident energies. The nitrogen sputtering decreases with growing energy. The reason for this is that at higher energies, nitrogen accumulates deeper in the material. Thus the concentration of nitrogen on the surface decreases. Hence fewer nitrogen atoms are in the surface available for the sputtering. However, the concentration of nitrogen increases with the number of cumulative runs. Actually, in the saturation case, which is described in the 3.2, the $\mathrm{N}$ sputtering yield reaches the value 1, fig. 7. The gradual growth of the $\mathrm{N}$ sputtering yield is expected for all energies when the system approaches saturation. The significant part of the accumulated nitrogen is sputtered as $\mathrm{N}_{2}$ molecules at low incidents energies, and the sputtering yield decreases sharply with an increase of energy, fig. 8. However, we observed the growth of the yield when enough nitrogen is retained. For example, at $100 \mathrm{eV}$ energy, the $\mathrm{N}_{2}$ sputtering yield increased by order of magnitude by the last part of the series of 2000 cumulative launches (the last calculated 500 runs). It reached the value 0.3 , which become comparable to the $10 \mathrm{eV}$ value. However, at $10 \mathrm{eV}$ energy, more than half of all sputtered nitrogen is in the molecular state from the very beginning of the simulation series, fig. 7. Slightly different sputtering mechanisms for 10 $\mathrm{eV}$ and higher investigated energies explain it.

The $\mathrm{N}_{2}$ molecule is volatile and binds weakly to the surface, and hence even low kinetic energy of the incoming particle is enough to sputter it. The primary condition of the $\mathrm{N}_{2}$ formation is the interaction time of the two particles. This time, and hence the relative velocity of two nitrogen atoms, has to be low enough for bond formation [30]. In the 10 $\mathrm{eV}$ case, the energy is low for the incident atoms to form a bond with nitrogen atoms on the surface itself. At higher energies, however, $\mathrm{N}_{2}$ forms usually when incident nitrogen collides with the $\mathrm{N}$ atom in the irradiated structure, and then the recoiled atom from 
the sample may interact with the $\mathrm{N}$ atom close to the surface and unite into molecule moving in the direction of the first recoiled atom. In this case, a significant concentration of nitrogen in the sample is another necessary condition for this type of sputtering.

Concluding the topic, we have to mention that the $\mathrm{N}_{2}$ sputtering yield growth is connected to the compositional changes in the surface layers. The comparison of the output sample after the simulation series for the energies up to $100 \mathrm{eV}$ showed that drastic changes of the $\mathrm{N}_{2}$ yield take place along with the slowdown of the $\mathrm{N}$ retention. By this time, the surface becomes extremely rough, and there is a large amount of nitrogen, weakly bound to it. Few percents limit the $\mathrm{N}_{2}$ sputtering yield for the higher energies during all the simulation. There are also no signs of the saturation at these cases, fig. 5 . However, a slight $\mathrm{N}_{2}$ yield increase is noticeable even in this case. We assume that this value will gradually become comparable with the yields at low energies.

Approximately half of the tungsten is sputtered in the mononitride molecule state at 300, 400, and $500 \mathrm{eV}$ energies, fig. 10. We have found significant WN sputtering in all cases when the notable number of tungsten is sputtered. This observation suggests that the same result will be observed at lower energies.

There are two mechanisms of the WN sputtering. The first scenario occurs when an incident or a recoil atom collides with tungsten atom at the very surface. The tungsten atom starts to move outside of the material, creating a vacancy in it. If there is a nitrogen interstitial near this area, it begins to move to the vacant space. The bond forms between the leaving tungsten atom and the nitrogen interstitial after that. Tungsten pulls nitrogen interstitial atom, and the WN molecule leaves the material. This can be a variety of the "dimer physical sputtering mechanism" [31,32]. In the second scenario, a molecule has formed at the surface by previous irradiation events and then can be physically sputtered in linear collision cascades. The recoil atom breaks the bond between the WN molecule and the material and does not directly transfer a major amount of kinetic energy. It is possible by the swift chemical sputtering (SCS) mechanism [33,34]. The same mechanism war reported previously for e.g. $\mathrm{BeH}, \mathrm{BeD}$, and $\mathrm{CH}$ molecules from initially pristine $\mathrm{Be}$, $\mathrm{W}$ and WC samples, respectively [35-37]. 


\section{Conclusions}

By means of molecular dynamic simulations, we studied the material properties changes in tungsten under prolonged nitrogen irradiation with different incident energies relevant to the condition of power plants: $10-1000 \mathrm{eV}$. In this study, we employed a newly developed WN potential based on Tersoff - Brenner formalism. The good agreement with experimental data gives us confidence that the applied interatomic potential can accurately describe the processes leading to the sputtering of the material.

With fluence increase, we observed a significant drop of an order of magnitude in the tungsten sputtering yield. This is explained by accumulation of nitrogen atoms in the surface layers and by the formation of W-N chemical bonds. The chemical bonds affect the surface cohesive energy of tungsten atoms in the material, which plays a significant role on sputtering at low incident energies.

We also observe the nitrogen saturation in the tungsten surface, which we explain by the steady growth of the nitrogen sputtering yield with the fluence. When the amount of nitrogen becomes sufficient to form dimers, the evaporation of molecular nitrogen (sputtering at very low, close to thermal, values of kinetic energies) contributes to the sputtering process leading faster to establishing the dynamic equilibrium of incoming and outgassing nitrogen during the bombardment. This factor plays a substantial role in the nitrogen saturation in the tungsten surface. The higher the energy, the deeper the nitrogen atoms penetrate, and hence the later the nitrogen saturation in the tungsten surface is reached. We also showed that the reflection coefficient does not affect the nitrogen saturation as it remains at approximately constant value $(\approx 0.3)$ for all of the studied energies.

In this work, we were able for the first time to identify the composition of the sputtered particles during the prolonged nitrogen bombardment of tungsten. We found that not only $\mathrm{N}_{2}$ dimers are forming at low energies $(\leq 100 \mathrm{eV})$, but also approximately half of sputtered tungsten atoms leave the surface in the state of a mononitride molecule at 300, 400 and $500 \mathrm{eV}$ energies. We showed that bond formation plays a significant role in molecular sputtering. In particular, the formation of $\mathrm{N}-\mathrm{N}$ bonds is essential for $\mathrm{N}_{2}$ sputtering, as is the formation of $\mathrm{W}-\mathrm{N}$ bonds for $\mathrm{WN}$ sputtering. In addition, the swift chemical sputtering mechanism was observed for the WN case. The total W sputtering yield agrees well with experimental data.

\section{Acknowledgements}

This work has been partly carried out within the framework of the EUROfusion Consortium and has received funding from the Euratom research and training programme 2014-2018 under grant agreement No 633053. The views and opinions expressed herein do not necessarily reflect those of the European Commission. 


\section{Data availability}

The raw and processed data required to reproduce these findings cannot be shared at this time due to time limitations. Please contact the authors directly for the data. 


\section{References}

[1] R. A. Pitts, S. Carpentier, F. Escourbiac, T. Hirai, V. Komarov, S. Lisgo, A. S. Kukushkin, A. Loarte, M. Merola, A. S. Naik, R. Mitteau, M. Sugihara, B. Bazylev, and P. C. Stangeby, "A full tungsten divertor for ITER : Physics issues and design status," Journal of Nuclear Materials, vol. 438, pp. S48-S56, 2013. [Online]. Available: http://dx.doi.org/10.1016/j.jnucmat.2013.01.008

[2] G. Federici, C. Skinner, J. Brooks, J. Coad, C. Grisolia, A. Haasz, A. Hassanein, V. Philipps, C. Pitcher, J. Roth, W. Wampler, and D. Whyte, "Plasma-material interactions in current tokamaks and their implications for next step fusion reactors," Nuclear Fusion, vol. 41, no. 12, pp. 1967-2137, dec 2001. [Online]. Available: https://doi.org/10.1088\%2F0029-5515\%2F41\%2F12\%2F 218

[3] G. Pacher, H. Pacher, G. Janeschitz, A. Kukushkin, V. Kotov, and D. Reiter, "Modelling of DEMO core plasma consistent with SOL/divertor simulations for long-pulse scenarios with impurity seeding," Nuclear Fusion, vol. 47, no. 5, pp. 469-478, may 2007. [Online]. Available: https://doi.org/10.1088\%2F0029-5515\% $2 \mathrm{~F} 47 \% 2 \mathrm{~F} 5 \% 2 \mathrm{~F} 012$

[4] K. Tobita, S. Nishio, M. Enoeda, H. Kawashima, G. Kurita, H. Tanigawa, H. Nakamura, M. Honda, A. Saito, S. Sato, T. Hayashi, N. Asakura, S. Sakurai, T. Nishitani, T. Ozeki, M. Ando, K. Ezato, K. Hamamatsu, T. Hirose, T. Hoshino, S. Ide, T. Inoue, T. Isono, C. Liu, S. Kakudate, Y. Kawamura, S. Mori, M. Nakamichi, H. Nishi, T. Nozawa, K. Ochiai, H. Ogiwara, N. Oyama, K. Sakamoto, Y. Sakamoto, Y. Seki, Y. Shibama, K. Shimizu, S. Suzuki, K. Takahashi, H. Tanigawa, D. Tsuru, T. Yamanishi, and T. Yoshida, "Compact DEMO, SlimCS: design progress and issues," Nuclear Fusion, vol. 49, no. 7, p. 075029, jul 2009. [Online]. Available: https://doi.org/10.1088\%2F0029-5515\%2F49\%2F7\%2F075029

[5] X. Bonnin, R. Pitts, V. Komarov, F. Escourbiac, M. Merola, L. Bo, L. Wei, L. Pan, and A. Kukushkin, "Iter divertor plasma response to time-dependent impurity injection," Nuclear Materials and Energy, vol. 12, pp. 1100-1105, 2017. [Online]. Available: https://doi.org/10.1016/j.nme.2017.03.010

[6] A. Kallenbach, R. Dux, M. Mayer, R. Neu, T. Pütterich, V. Bobkov, J. Fuchs, T. Eich, L. Giannone, O. Gruber, A. Herrmann, L. Horton, C. Maggi, H. Meister, H. Müller, V. Rohde, A. Sips, A. Stäbler, and J. S. and, "Non-boronized compared with boronized operation of ASDEX upgrade with full-tungsten plasma facing components," Nuclear Fusion, vol. 49, no. 4, p. 045007, mar 2009. [Online]. Available: https://doi.org/10.1088\%2F0029-5515\%2F49\%2F4\%2F045007 
[7] A. Kallenbach, M. Balden, R. Dux, T. Eich, C. Giroud, A. Huber, G. P. Maddison, M. Mayer, K. McCormick, R. Neu, T. W. Petrie, T. Pütterich, J. Rapp, M. L. Reinke, K. Schmid, J. Schweinzer, and S. Wolfe, "Plasma surface interactions in impurity seeded plasmas," Journal of Nuclear Materials, vol. 415, no. 1 SUPPL, pp. S19-S26, 2011. [Online]. Available: http://dx.doi.org/10.1016/j.jnucmat.2010.11.105

[8] K. Schmid, A. Manhard, C. Linsmeier, A. Wiltner, T. Schwarz-Selinger, W. Jacob, and S. Mändl, "Interaction of nitrogen plasmas with tungsten," Nuclear Fusion, vol. 50, no. 2, p. 025006, 2010. [Online]. Available: http://stacks.iop.org/0029-5515/ $50 / \mathrm{i}=2 / \mathrm{a}=025006 ? \mathrm{key}=$ crossref.aa589c1153f4c2d114a12a31da37adfc

[9] G. W. Samsonov, Nitridi (Nitrides in Russian). Kiev: Naukova Dumka, 1969.

[10] A. N. Krestovnikov and A. S. Shahow, "Fiziko-khimicheskiye i termodinamicheskiye svoystva redkikh elementov (Physico-chemical and thermodynamic properties of rare elements in Russian)," Metallurgizdat, 1939.

[11] Gmelins, Gmelin Handbuch Wolfram (Tungsten Handbook in German), Berlin, 1933.

[12] T.-c. Software, "THERMO-CALC \& DICTRA, Computational Tools For Materials Science J-O Andersson, Thomas Helander,Lars Hdghmd, Pingfang Shi, Bo Sundman," Calphad Computer Coupling Of Phase Diagrams And Thermochemistry, 2002. [Online]. Available: https://doi.org/10.1016/S0364-5916(02)00037-8

[13] O. V. Ogorodnikova, K. Sugiyama, A. Markin, Y. Gasparyan, V. Efimov, A. Manhard, and M. Balden, "Effect of nitrogen seeding into deuterium plasma on deuterium retention in tungsten," Physica Scripta, vol. T145, p. 014034, 2011. [Online]. Available: http://stacks.iop.org/1402-4896/2011/i=T145/a=014034?key= crossref.e3d0c5fdfc410776a07f4be486ce484f

[14] G. Meisl, K. Schmid, O. Encke, T. Höschen, L. Gao, and C. Linsmeier, "Implantation and erosion of nitrogen in tungsten," New Journal of Physics, vol. 16, no. 9, p. 093018, sep 2014. [Online]. Available: https://doi.org/10.1088\% 2F1367-2630\%2F16\%2F9\%2F093018

[15] R. Frauenfelder, "Permeation of Hydrogen through Tungsten and Molybdenum," The Journal of Chemical Physics, vol. 48, no. 9, p. 3955, 1968. [Online]. Available: http://scitation.aip.org/content/aip/journal/jcp/48/9/10.1063/1.1669720

[16] J. Keinonen, J. Räisänen, and A. Anttila, "Diffusion of Nitrogen in Ion-Implanted Chromium and Tungsten," Applied Physics A Solids and Surfaces, vol. 35, no. 4, pp. 227-232, 1984. [Online]. Available: http://www.springerlink.com/index/10.1007/ BF00617172 
[17] P. Petersson, A. Hakola, J. Likonen, M. Mayer, J. Miettunen, R. Neu, V. Rohde, and M. Rubel, "Injection of nitrogen-15 tracer into ASDEX-Upgrade: New technique in material migration studies," Journal of Nuclear Materials, 2013. [Online]. Available: https://doi.org/10.1016/j.jnucmat.2013.01.129

[18] A. Kallenbach, R. Dux, J. C. Fuchs, R. Fischer, B. Geiger, L. Giannone, A. Herrmann, T. Lunt, V. Mertens, R. McDermott, R. Neu, T. Pütterich, S. Rathgeber, V. Rohde, K. Schmid, J. Schweinzer, and W. Treutterer, "Divertor power load feedback with nitrogen seeding in ASDEX Upgrade," Plasma Physics and Controlled Fusion, vol. 52, no. 5, p. 055002, 2010. [Online]. Available: http://stacks.iop.org/0741-3335/ $52 / \mathrm{i}=5 / \mathrm{a}=055002$ ?key $=$ crossref.cc7cc88ca20e822dd0724980a12286fb

[19] W. Eckstein, Computer simulation of ion-solid interactions. Springer Science \& Business Media, 2013, vol. 10. [Online]. Available: https://doi.org/10.1007/ 978-3-642-73513-4

[20] J. Polvi, K. Heinola, and K. Nordlund, "An interatomic potential for W?N interactions," Modelling and Simulation in Materials Science and Engineering, vol. 24, no. 6, p. 065007, 2016. [Online]. Available: http://stacks.iop.org/0965-0393/ 24/i=6/a=065007?key=crossref.6d7c4babad82a61099dec58091a41b7a

[21] J. Tersoff, "New empirical approach for the structure and energy of covalent systems," Physical Review B, vol. 37, no. 12, p. 6991, 1988. [Online]. Available: https://doi.org/10.1103/PhysRevB.37.6991

[22] D. W. Brenner, "Empirical potential for hydrocarbons for use in simulating the chemical vapor deposition of diamond films," Physical review B, vol. 42, no. 15, p. 9458, 1990. [Online]. Available: https://doi.org/10.1103/PhysRevB.42.9458

[23] C. Björkas, K. O. E. Henriksson, M. Probst, and K. Nordlund, "A be-w interatomic potential," Journal of Physics: Condensed Matter, vol. 22, no. 35, p. 352206 , aug 2010. [Online]. Available: https://doi.org/10.1088\%2F0953-8984\%2F22\%2F35\% 2F352206

[24] N. Juslin, P. Erhart, P. Träskelin, J. Nord, K. O. Henriksson, K. Nordlund, E. Salonen, and K. Albe, "Analytical interatomic potential for modeling nonequilibrium processes in the $\mathrm{w}-\mathrm{c}-\mathrm{h}$ system," Journal of applied physics, vol. 98, no. 12, p. 123520, 2005. [Online]. Available: https://doi.org/10.1063/1.2149492

[25] H. J. C. Berendsen, J. P. M. Postma, W. F. van Gunsteren, A. DiNola, and J. R. Haak, "Molecular dynamics with coupling to an external bath," The Journal of Chemical Physics, vol. 81, no. 8, pp. 3684-3690, 1984. [Online]. Available: http://aip.scitation.org/doi/10.1063/1.448118 
[26] K. Nordlund, F. Djurabekova, and G. Hobler, "Large fraction of crystal directions leads to ion channeling," Phys. Rev. B, vol. 94, p. 214109, Dec 2016. [Online]. Available: https://link.aps.org/doi/10.1103/PhysRevB.94.214109

[27] J. Nord, K. Nordlund, and J. Keinonen, "Amorphization mechanism and defect structures in ion-beam-amorphized si, ge, and gaas," Phys. Rev. B, vol. 65, p. 165329, Apr 2002. [Online]. Available: https://link.aps.org/doi/10.1103/PhysRevB. 65.165329

[28] K. Nordlund, "Parcas computer code," 2006.

[29] R. Behrisch and W. Eckstein, Sputtering by particle bombardment: experiments and computer calculations from threshold to $\mathrm{MeV}$ energies. Springer Science \& Business Media, 2007, vol. 110. [Online]. Available: https://doi.org/10.1007/ 978-3-540-44502-9

[30] A. V. Krasheninnikov, K. Nordlund, E. Salonen, J. Keinonen, and C. H. Wu, "Sputtering of amorphous hydrogenated carbon by hyperthermal ions as studied by tight-binding molecular dynamics," Comput. Mater. Sci, vol. 25, pp. 427-434, 2002. [Online]. Available: https://doi.org/10.1016/S0927-0256(02)00318-X

[31] K. Vörtler, C. Björkas, and K. Nordlund, "The effect of plasma impurities on the sputtering of tungsten carbide," J. Phys.: Conden. Matter, vol. 23, no. 8, p. 085002 , 2010. [Online]. Available: http://stacks.iop.org/0953-8984/23/i=8/a=085002

[32] F. Karetta and H. M. Urbassek, "The dimer sputtering mechanism of cu(001) at low bombarding energy." Appl. Phys. A, vol. 55, pp. 364-371, 1992. [Online]. Available: https://doi.org/10.1007/BF00324086

[33] E. Salonen, K. Nordlund, J. Keinonen, and C. H. Wu, "Swift chemical sputtering of amorphous hydrogenated carbon," Phys. Rev. B, vol. 63, p. 195415, Apr 2001. [Online]. Available: https://link.aps.org/doi/10.1103/PhysRevB.63.195415

[34] K. Nordlund, C. Björkas, K. Vörtler, A. Meinander, A. Lasa, M. Mehine, and A. V. Krasheninnikov, "Mechanism of swift chemical sputtering: comparison of Be/C/W dimer bond breaking," Nucl. Instr. Meth. Phys. Res. B, vol. 269, no. 11, pp. 1257-1261, 2011. [Online]. Available: http://dx.doi.org/10.1016/j.nimb.2010.12.080

[35] C. Björkas, K. Vörtler, K. Nordlund, D. Nishijima, and R. Doerner, "Chemical sputtering of be due to d bombardment," New Journal of Physics, vol. 11, no. 12, p. 123017, dec 2009. [Online]. Available: https: //doi.org/10.1088\%2F1367-2630\%2F11\%2F12\%2F123017 
[36] A. Lasa, K. Heinola, and K. Nordlund, "Atomistic simulations of be irradiation on w: mixed layer formation and erosion," Nuclear Fusion, vol. 54, no. 8, p. 083001, may 2014. [Online]. Available: https://doi.org/10.1088\%2F0029-5515\%2F54\%2F8\% 2F083001

[37] A. Lasa, C. Björkas, K. Vörtler, and K. Nordlund, "MD Simulations of Low Energy Deuterium Irradiation on W, WC andW2C surfaces," J. Nucl. Mater., vol. 429, pp. 284-292, 2012. [Online]. Available: https://doi.org/10.1016/j.jnucmat.2012.06.012

[38] Möller, Wolfhard and Eckstein, Wolfgang and Biersack, JP, "Tridyn-binary collision simulation of atomic collisions and dynamic composition changes in solids," Computer Physics Communications, vol. 51, pp. 355-368, 1988. [Online]. Available: https://doi.org/10.1016/0010-4655(88)90148-8

\section{Figures}

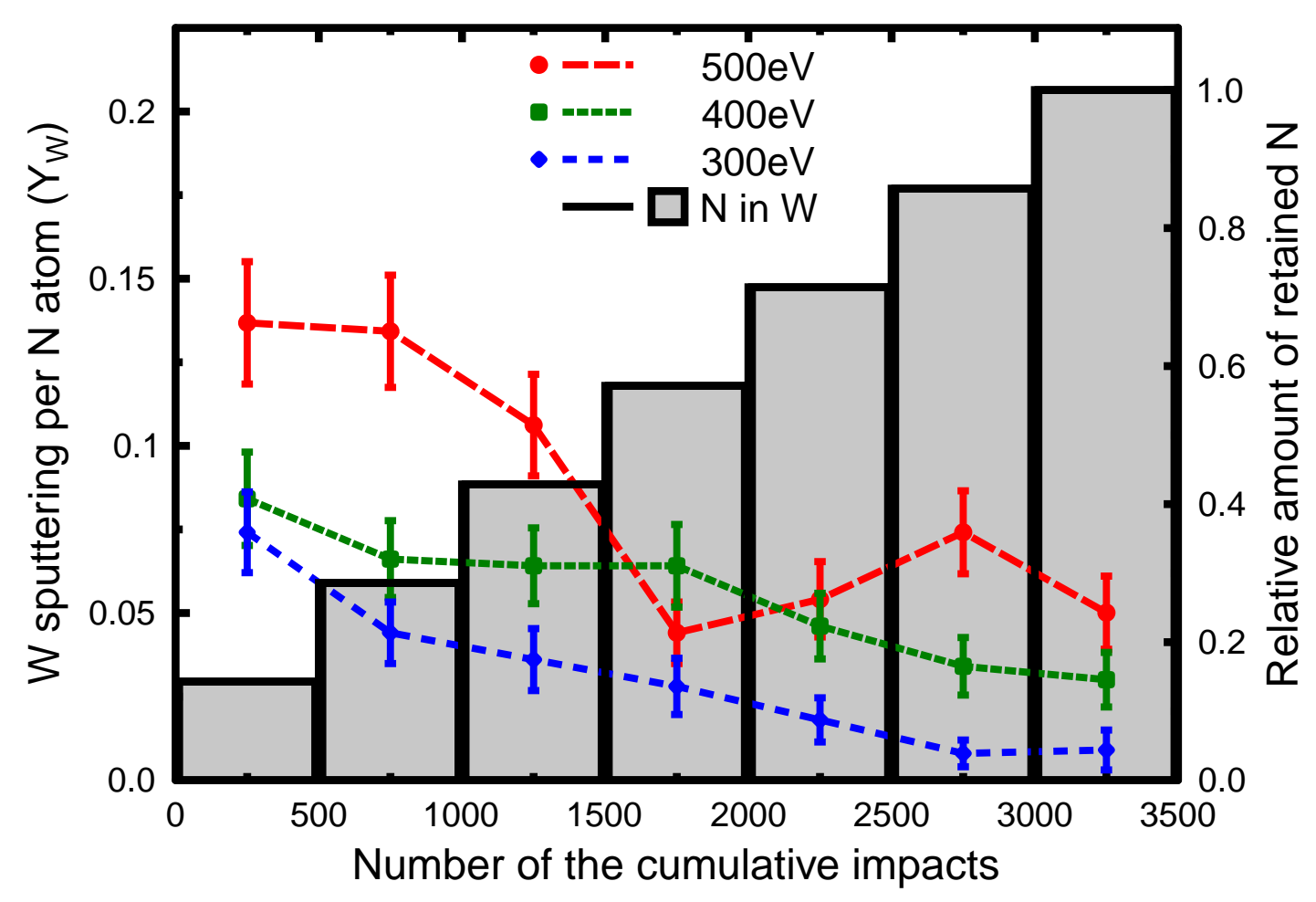

Figure 1. The dependence of the tungsten sputtering yield on nitrogen fluence at 300(blue), 400 (green) and 500 (red) eV nitrogen energies. The gray bars show the relative nitrogen fluence. In absolute numbers the maximum fluence is $4.8 \cdot 10^{19} \mathrm{~m}^{-2}$. 


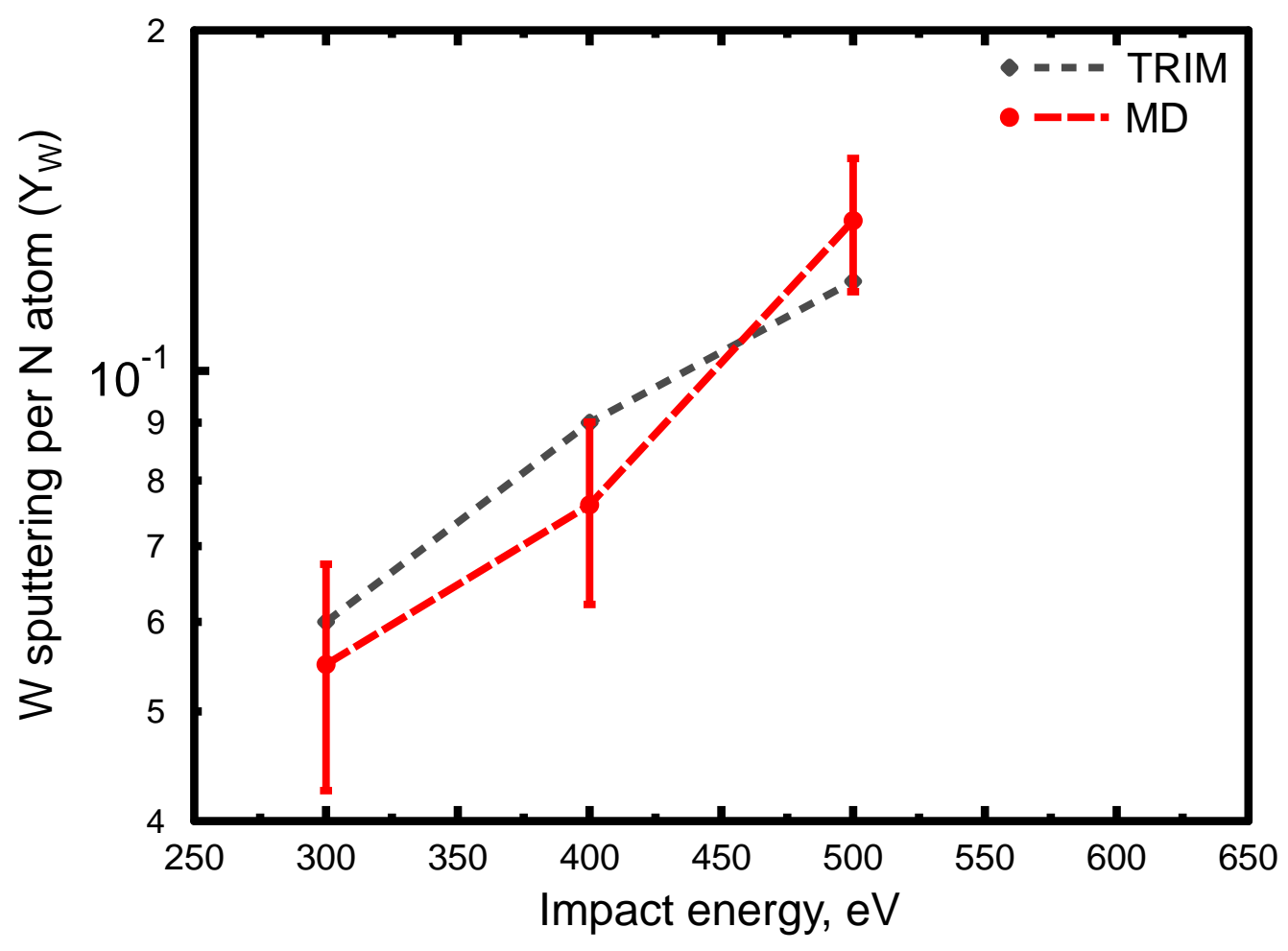

Figure 2. The tungsten sputtering yield dependence on incident energy. The sputtering yields calculated by the two methods: TRIM (BCA) and MD. We averaged the molecular dynamic calculation over first 1-1.5 thousand of cumulative impacts since the accumulated amount of nitrogen in the tungsten lattice by this fluence is not yet sufficient to affect notably the sputtering yield of tungsten. Molecular dynamics agrees well with the BCA results. 


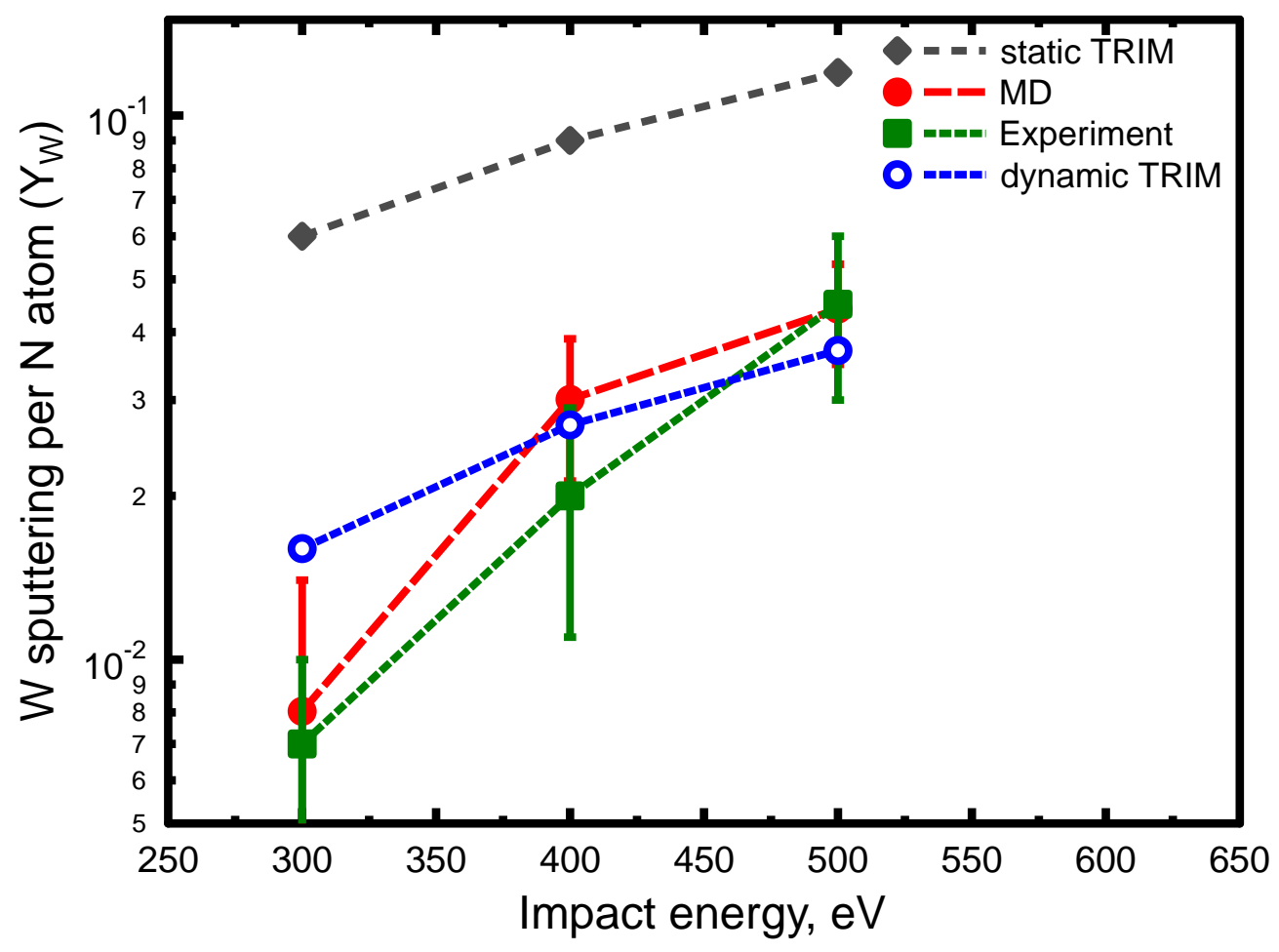

Figure 3. The dependence of the tungsten sputtering yield on energies of the incoming nitrogen atoms. Molecular dynamics results were averaged over the remainder of the simulation runs, after the yield reaches the constant value. This happens after about 2.5-3.5 thousand of impacts, which correspond to a fluence of $3.4-4.8 \cdot 10^{19}$. 


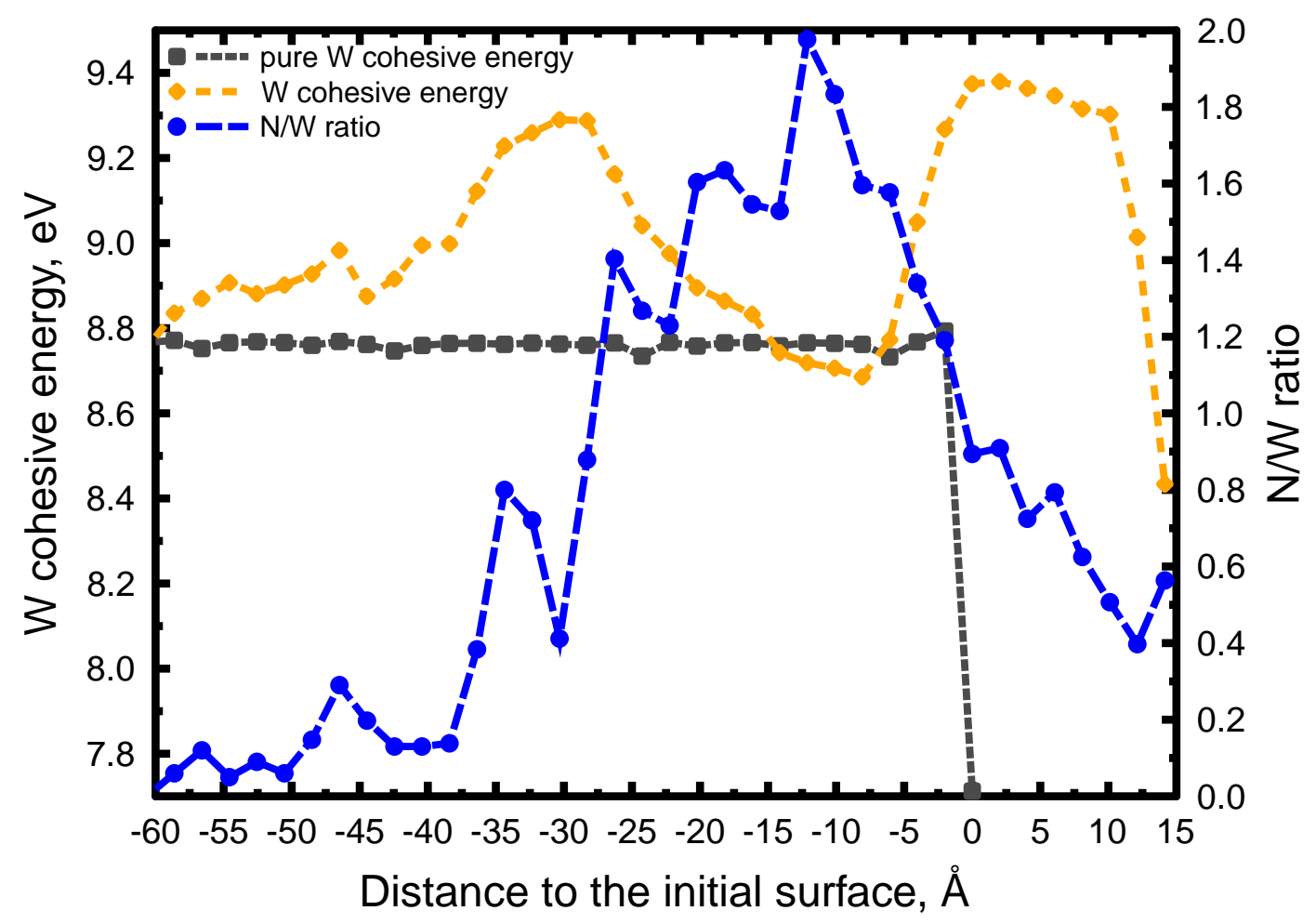

Figure 4. Depth profiles of the tungsten cohesive energy and the $\frac{N_{N}}{N_{W}}$ ratio with respect to the initial surface level for $300 \mathrm{eV}$ case. In this graph, the cohesive energies of $\mathrm{W}$ atoms as well as the $\frac{N_{N}}{N_{W}}$ ratios were obtained by averaging these values for all corresponding atoms found in the $2 \AA$ slabs dividing the simulation cell perpendicular to the z-axis. An interval from 0 to $15 \AA$ corresponds to the expanded part of the sample, resulting from nitrogen accumulation. 


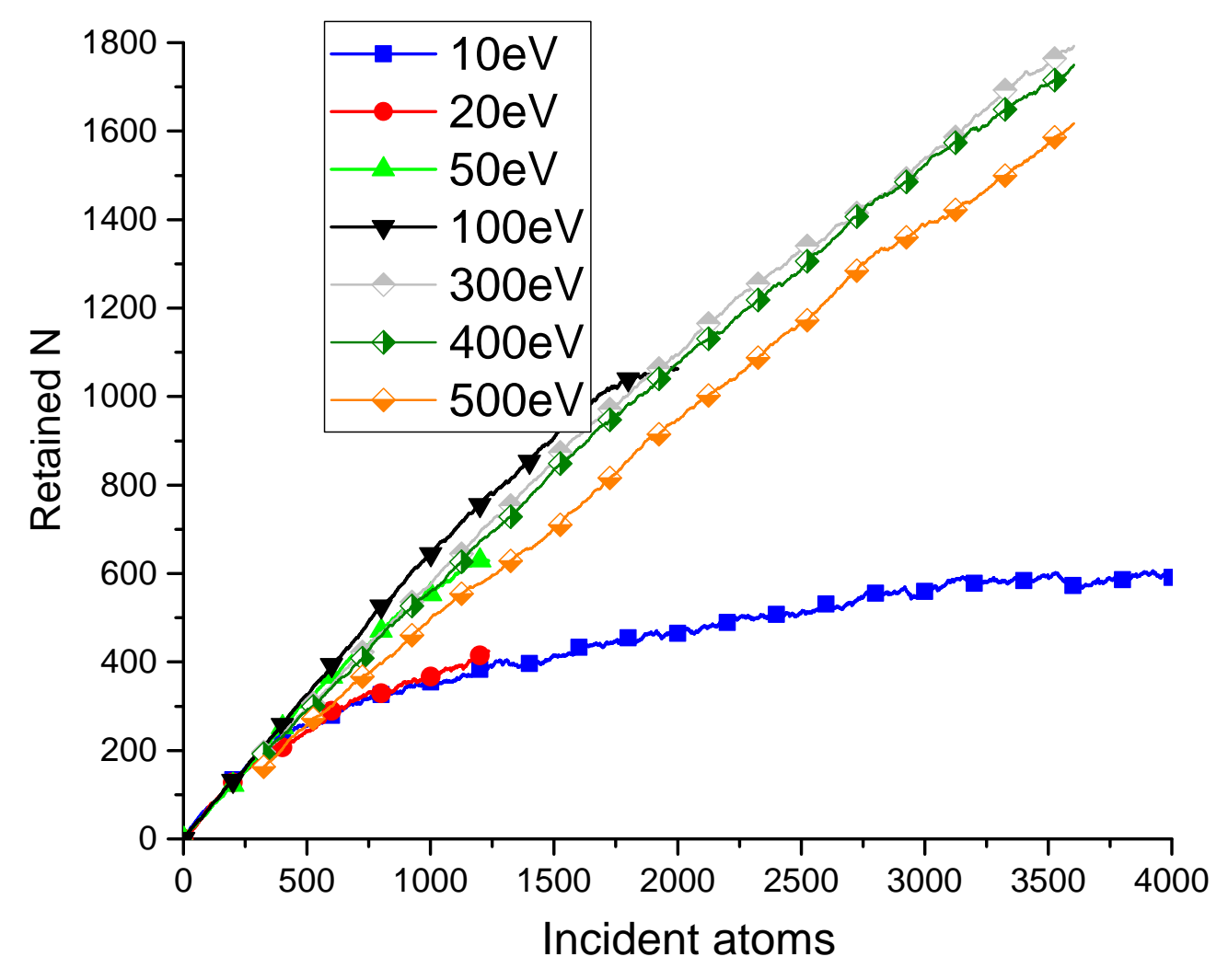

Figure 5. The dependence of the amount of nitrogen retained in the material on the number of incident atoms for various energies. 3,5 thousand impacts correspond to a fluence of $4.8 \cdot 10^{19} \mathrm{~m}^{-2}$. For an energy of $10 \mathrm{eV}, 6095$ collisions of nitrogen with tungsten were simulated (only 4000 are shown on the graph). The complete dependence is shown in fig. 7 . 


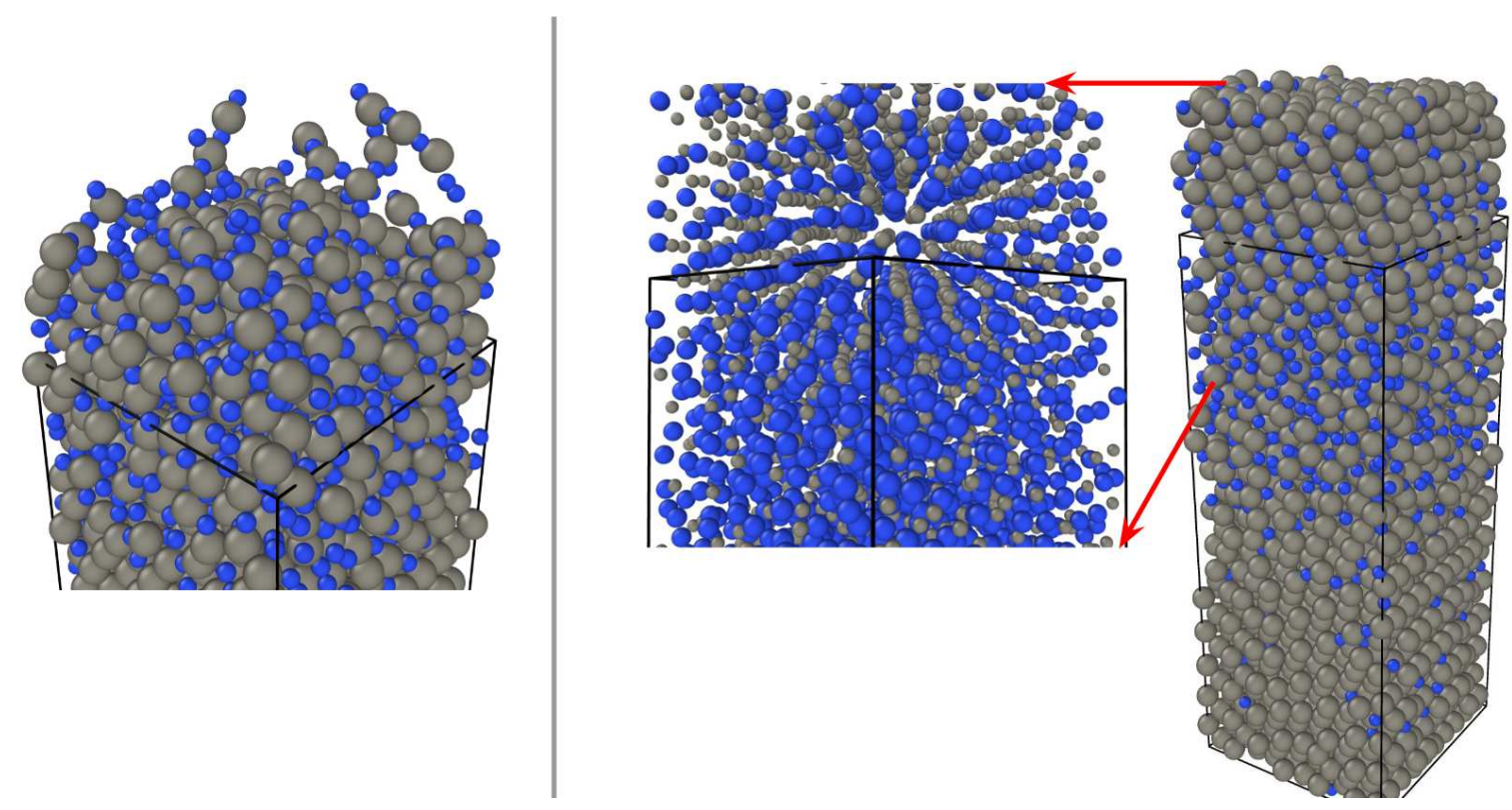

Figure 6. The surface morphology of $\mathrm{W}$ cells after 2000 impacts at $100 \mathrm{eV}$ energy(left) and 3380 impacts at $300 \mathrm{eV}$ energy (right). The gray spheres represent $\mathrm{W}$ atoms, the blue spheres $\mathrm{N}$ atoms. The black box shows the initial sample size. Red arrows indicate a different view of the same sample $(300 \mathrm{eV})$ in the right part of the figure. The proportions of tungsten and nitrogen atoms are altered to emphasize the structure orientation above the initial surface layer. 


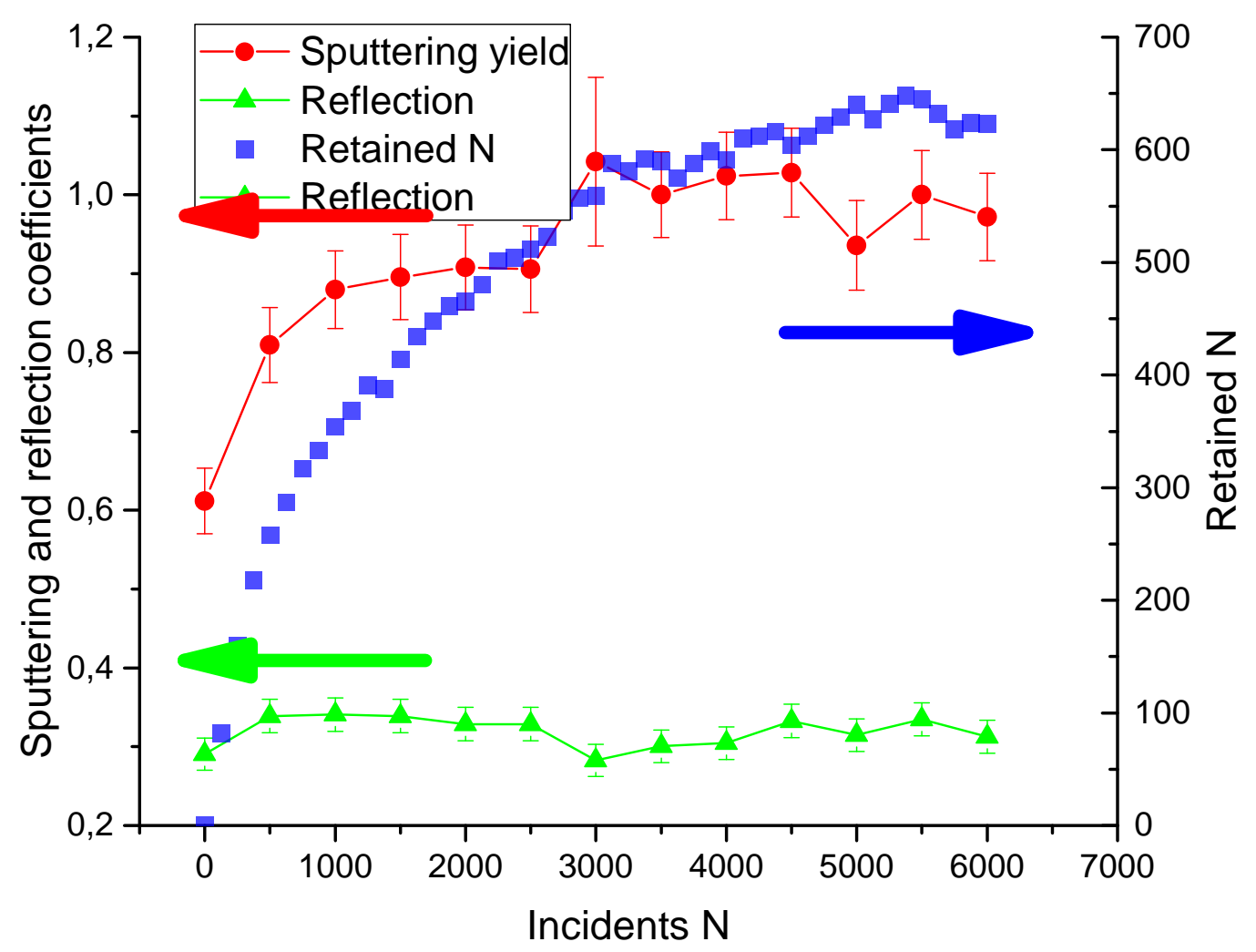

Figure 7. Evolution of sputtering yield, reflection coefficient and retained amount of nitrogen with the fluence during the $10 \mathrm{eV}$ nitrogen irradiation of tungsten. Right Y-axis shows the amount of the nitrogen retained in the material (blue squares) for the $10 \mathrm{eV}$ bombardment. The left Y-axis shows the sputtering yield (red circles) and the reflection (green triangles) coefficient. 6095 impacts is shown in the figure, it corresponds to a fluence of $8.4 \cdot 10^{19} \mathrm{~m}^{-2}$. 


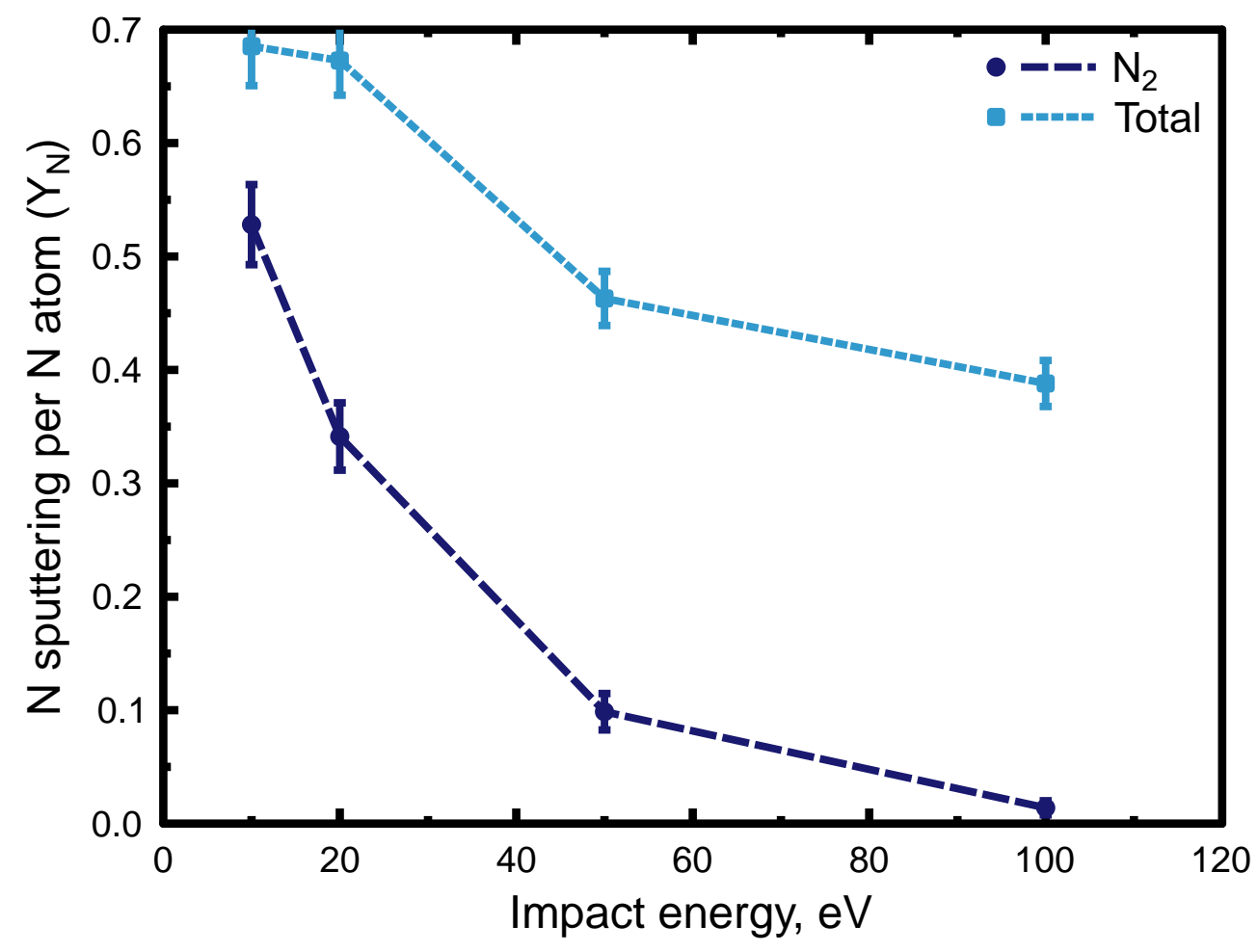

Figure 8. The dependence of the total and of the molecular nitrogen sputtering yield on incident energy. The yields were calculated after 1200 cumulative runs for all energies (fluence $1.6 \cdot 10^{19} \mathrm{~m}^{-2}$ ). 


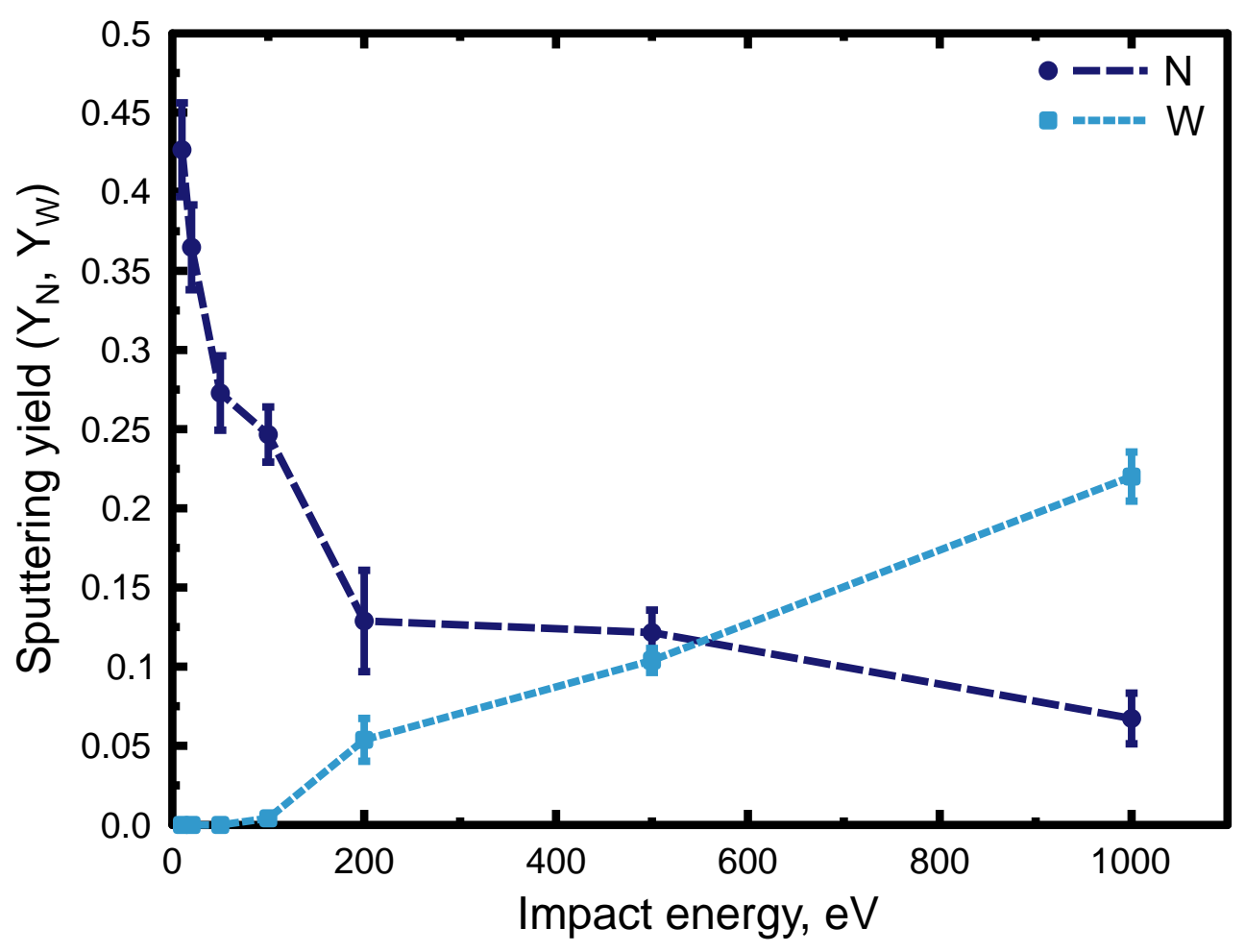

Figure 9. The dependence of the nitrogen and of the tungsten sputtering yields separately on incident energies. The yields were achieved after 1200 impacts for all energies (fluence $1.6 \cdot 10^{19} \mathrm{~m}^{-2}$ ). 


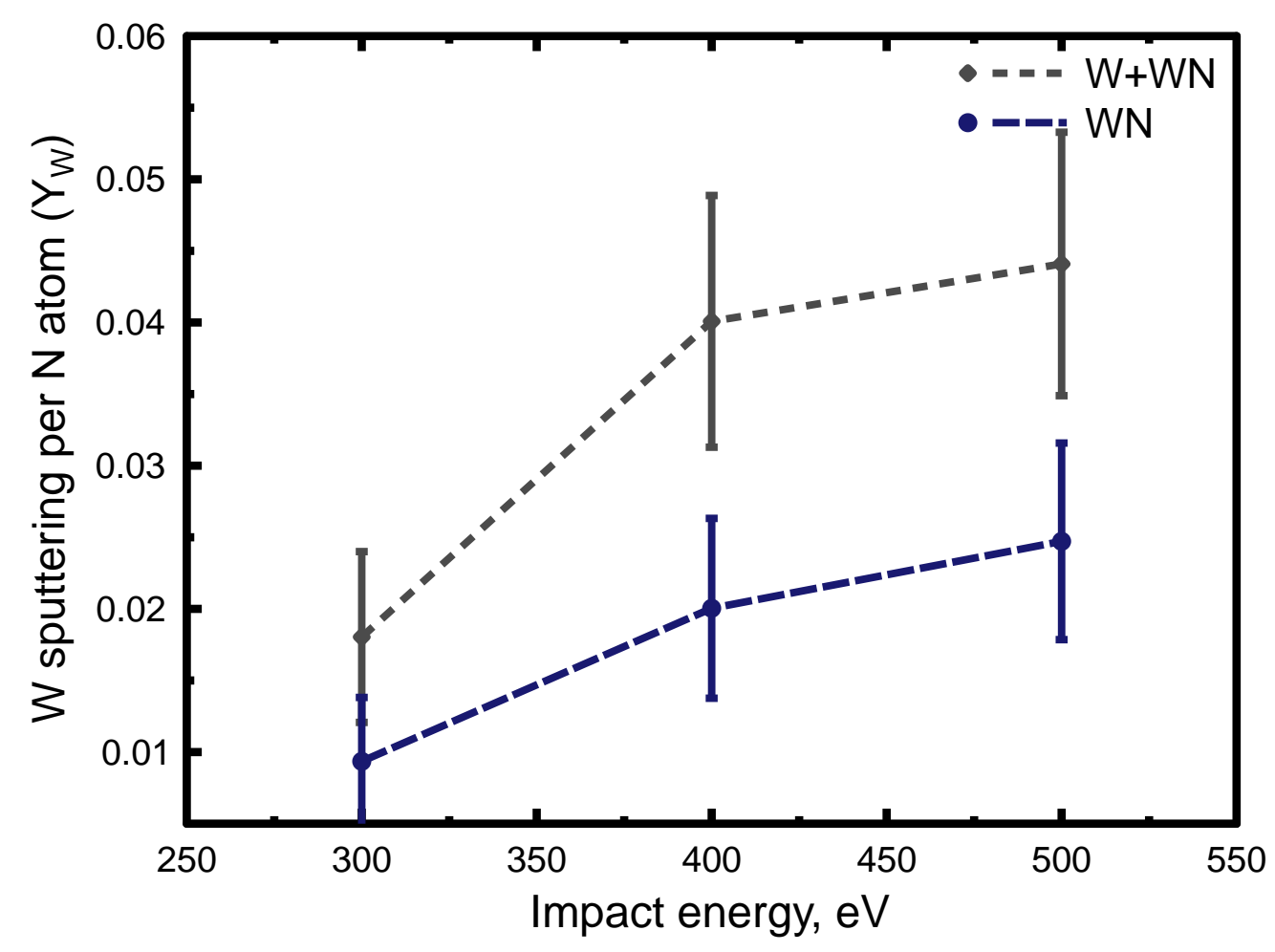

Figure 10. The dependence of the mononitride (WN) compound and of the total tungsten (W) sputtering yield on incident energy. These values were calculated after 3500 cumulative simulation for all energies (fluence $4.8 \cdot 10^{19} \mathrm{~m}^{-2}$ ). 